\title{
Biphasic Type of Malignant Mesothelioma Arising in the Peritoneum - A Case Report
}

\author{
Syed Fiza Mustaqueem ${ }^{1}$, Pranab Kumar Vermaㄹ Mona Lisa $^{3}$, Nishi ${ }^{4}$, Md. Anwar Hussain ${ }^{5}$ \\ ${ }^{1}$ Department of Pathology, Mahavir Cancer Sansthan, Patna, Bihar, India, ${ }^{2}$ Department of \\ Pathology, Mahavir Cancer Sansthan, Patna, Bihar, India, ${ }^{3}$ Department of Pathology, Mahavir \\ Cancer Sansthan, Patna, Bihar, India, ${ }^{4}$ Department of Pathology, Mahavir Cancer Sansthan, Patna, \\ Bihar, India, ${ }^{5}$ Department of Pathology, Mahavir Cancer Sansthan, Patna, Bihar, India.
}

\section{INTRODUCTION}

Malignant Peritoneal Mesothelioma (MPM) is a rare aggressive tumour of the peritoneum with a rapid fatal outcome.(1) It is usually diagnosed in the advanced stages in most cases and it often takes considerable time to reach the correct diagnosis.(2) In this study, we are reporting a case of biphasic type of malignant mesothelioma arising in the peritoneum in a 70 year old male patient. The tumour was resected, and histopathological examination and immunohistochemistry was done to reach at the diagnosis. We are presenting this case to highlight that although rare, we should consider it in the differential diagnosis of any mass arising from peritoneum so that multimodality treatment can be planned accordingly, and a relatively longer survival can be expected.

Most of the cases of MPM arise from pleura (65-70\%), followed by peritoneum (30\%), tunica vaginalis of testis, and pericardium.(1) MPM of peritoneum is an aggressive neoplasm with a rapidly fatal course. It should always be thought of as a differential in retroperitoneal tumours and immunohistochemistry plays an important role in the diagnosis.

\section{PRESENTATION OF CASE}

A 70 - year old male with a history of tobacco chewing presented with a painful mass in the lower abdomen and dribbling of urine for a period of six months. On physical examination, a painful and immobile mass was felt in lower abdomen measuring approximately $15 \mathrm{~cm}$ in diameter. All the clinical parameters were within normal ranges. Computed tomography (CT) showed a large, heterogeneously enhancing well defined mass measuring $14.1 \times 13.9 \times 17.8 \mathrm{~mm}$ in the right lower abdomen extending from lower retro peritoneum just above the aortic bifurcation into the pelvis. Sub centimetre retroperitoneal and iliac inguinal lymph nodes are identified. The radiological impression was that of liposarcoma. Wide local excision of the mass and reconstruction of the peritoneum was done. On observing the thoracic and peritoneal cavities, neither pleural nor peritoneal dissemination was found. The patient had an uneventful post-operative recovery without any complications.

Grossly the tumour was approximately $16 \mathrm{~cm}$ in diameter and was well encapsulated. On cut section, it was pale yellow in colour with foci of haemorrhage and necrosis. Histologically, the tumour showed biphasic pattern comprising of epithelioid and spindle cell components. The spindle cell component was moderately cellular and vascular with pleomorphic spindle cells showing scattered atypical mitoses. Bizarre tumour cells were also present.
Corresponding Author:

Mona Lisa,

302 B, Ramdeokunj, Anandpuri,

Patna-800001, Bihar, India.

E-mail:monaloud@gmail.com

DOI: $10.14260 / \mathrm{jemds} / 2020 / 539$

How to Cite This Article:

Mustaqueem SF, Verma PK, Lisa M, et al. Biphasic type of malignant mesothelioma arising in the peritoneum- report of a case. J Evolution Med Dent Sci 2020;9(34):2484 2486, DOI: 10.14260/jemds/2020/539

Submission 17-05-2020,

Peer Review 10-07-2020,

Acceptance 17-07-2020,

Published 24-08-2020.

Copyright (C) 2020 JEMDS. This is an open access article distributed under Creative Commons Attribution License [Attribution 4.0 International (CC BY 4.0)] 
It was reported as spindle cell neoplasm, giving dedifferentiated liposarcoma, leiomyosarcoma, malignant peripheral nerve sheath tumour and malignant mesothelioma as the differential diagnoses. Immunohistochemically the epithelial component of the tumour cells showed positive reaction to CK, EMA and CK 5 / 6; while CD 34 and D - 240 marked the spindle cells. These cells show focal expression of desmin. The tumour cells were immunonegative for S - 100 protein, SMA, calretinin and WT - 1 .

Fluorodeoxyglucose positron emission tomography (FDG PET) was performed 1 year after the surgery to find out any recurrence. FDG - PET showed multiple mildly FDG avid heterogeneously enhancing necrotic peritoneal soft tissue lesion in the abdominal cavity measuring approximately $7.5 \mathrm{x}$ $6.7 \times 4.5 \mathrm{~cm}$ likely to be metastatic deposits. The patient was given adjuvant chemotherapy and radiotherapy and was closely followed thereafter.
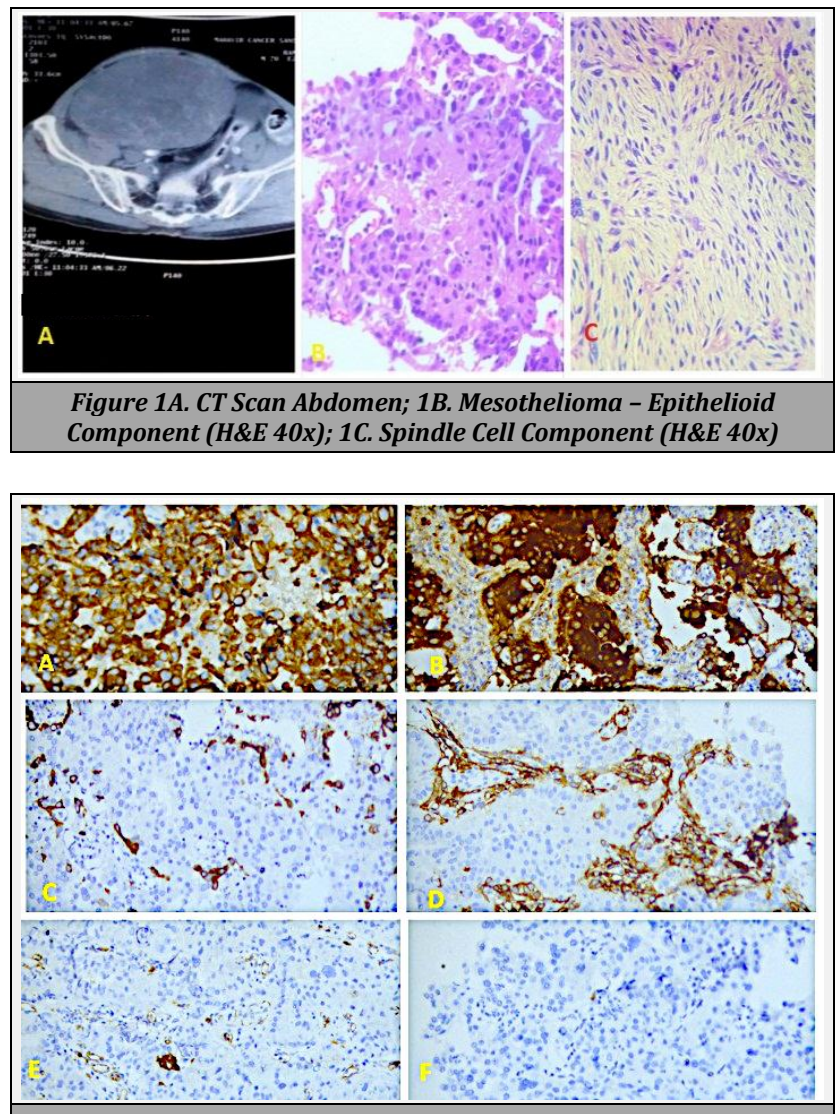

Figure 2A. CK Expression in the Epithelioid Component; 2B. EMA Expression in the Epithelioid Component; 2 C. CK 5/6 Expression in the Epithelioid Component; 2D. D240 Expression in the Spindle Cell Component; 2E. WT1 Not Expressed in Tumor Cells; $2 F$. Calretinin Not Expressed in Tumor Cells

\section{DISCUSSION}

MPM was first described in 1908 by Miller and Wynn. It is more common in males as compared to females with male: female ratio being approximately $2: 1$. The patients are usually middle aged or elderly, but occasional cases have been reported in young adults or children. Prior studies have shown that only $50 \%$ of patients with a peritoneal origin of tumour have a history of asbestos exposure.(3) In our case also, there was no history of exposure to asbestos.
MPM is classified into three histological subtypes epithelioid, sarcomatoid and biphasic. A biphasic tumour has both epithelioid and sarcomatous components, each of which contributes to more than $10 \%$ of overall histology. As per the data available, the epithelioid type is the most common.(4) Very few tumour having biphasic type have been reported.(5) And there has been no previous case report of the purely sarcomatoid type. The incidence of biphasic type of MPM is lower in peritoneum than in pleural disease.(6) The prognosis of epithelioid MPM is better than biphasic or sarcomatoid type. Study done by Sugarbaker et al has reported that median survival of the patients with epithelioid MPM is 55 months as compared to only 13 months for patients with combination of sarcomatoid and biphasic subtypes.(7)

Patients usually present with non-specific manifestations most commonly abdominal discomfort and distention, digestive disturbances and weight loss.(8) Diagnosis usually requires laparotomy or laparoscopy and biopsy.

Computed tomography (CT) findings of MPM are nonspecific and not sufficient to establish a diagnosis; however CT is useful for surgical planning and staging and guiding biopsy of peritoneal masses.(9) Therefore the definitive diagnosis of MPM depends on histologic and IHC examination.

A panel of IHC markers has been suggested for diagnostic aid. Most MPM are immunoreactive for CK 5 / 6 and calretinin lack reactivity for a variety of "epithelioid" antigens, the most useful of which are CEA, B72.3, CD 15 (LeuM1), Ber EP4, S 100 and placental alkaline phosphatase (PLAP). To differentiate MPM from serous adenocarcinoma, immunoreactivity for Ber EP4, B72.3, LeuM1, MOC 31 and CA - 19 - 9 favours serous carcinoma; whereas immunoreactivity for thrombomodulin, D2 - 40 (or podoplanin) and calretinin favours MPM.(10) No single IHC stain is diagnostic in separation of MPM from adenocarcinoma and the results of a panel of antibodies should be interpreted along with haematoxylin and eosin stain.

For patients with confirmed MPM, cytoreductive surgery followed by intraperitoneal hypothermic perfusion is the standard treatment for resectable tumour at diagnosis.(11) Data from studies suggest systemic chemotherapy to be the standard of care for patients with unresectable MPM.(12) Radiotherapy has only a limited role in MPM and is not currently used.(13) Biphasic type of MPM, although rare, is considered to be one of the most aggressive histotype. It is often difficult to diagnose this disease at an early stage because of the vague clinical presentations over a long time. But with early diagnosis and multimodality treatment approach a relatively long survival can be expected.

Financial or Other Competing Interests: None.

\section{REFERENCES}

[1] Cunha P, Luz Z, Seves I, et al. Malignant peritoneal mesothelioma--diagnostic and therapeutic difficulties. Acta Med Port 2002;15(5):383-6.

[2] de Pangher Manzini V. Malignant peritoneal mesothelioma. Tumori 2005;91(1):1-5. 
[3] Busch JM, Kruskal JB, Wu B. Best cases from the AFIP. Malignant peritoneal mesothelioma. Radiographics 2002;22(6):1511-5.

[4] Allen TC, Cagle PT, Churg AM, et al. Localized malignant mesothelioma. Am J Surg Pathol 2005;29(7):866-73.

[5] Matsukuma S, Aida S, Hata Y, et al. Localized malignant peritoneal mesothelioma containing rhabdoid cells. Pathol Int 1996;46(5):389-91.

[6] Husain AN, Colby T, Ordonez N, et al. Guidelines for pathologic diagnosis of malignant mesothelioma: 2012 update of the consensus statement from the International Mesothelioma Interest Group. Arch Pathol Lab Med 2013;137(5):647-67.

[7] Sugarbaker PH, Welch LS, Mohamed F, et al. A review of peritoneal mesothelioma at the Washington Cancer Institute. Surg Oncol Clin N Am 2003;12(3):605-21.

[8] Baker PM, Clement PB, Young RH. Malignant peritoneal mesothelioma in women: a study of 75 cases with emphasis on their morphologic spectrum and differential diagnosis. Am J Clin Pathol 2005;123(5):724-37.
[9] Pickhardt PJ, Bhalla S. Primary neoplasms of peritoneal and sub-peritoneal origin: CT findings. Radiographics 2005;25(4):983-95.

[10] Ordonez NG. The diagnostic utility of immunohistochemistry and electron microscopy in distinguishing between peritoneal mesothelioma and serous carcinoma: a comparative study. Mod Pathol 2006;19(1):34-48.

[11] Kusamura S, Younan R, Baratti D, et al. Cytoreductive surgery followed by intraperitoneal hyperthermic perfusion: analysis of morbidity and mortality in 209 peritoneal surface malignancies treated with closed abdomen technique. Cancer 2006;106(5):1144-53.

[12] Garcia-Carbonero R, Paz-Ares L. Systemic chemotherapy in the management of malignant peritoneal mesothelioma. Eur J Surg Oncol 2006;32(6):676-81.

[13] Lederman GS, Recht A, Herman T, et al. Long-term survival in peritoneal mesothelioma. The role of radiotherapy and combined modality treatment. Cancer 1987;59(11):1882-6. 